\title{
Proceedings
}

Juntendo Medical Journal

2016. 62 (Suppl 1), 22-28

\section{Does the Sports Gene Affect Lifestyle-Related Diseases?}

\author{
NORIYUKI FUKU*1), ERI MIYAMOTO-MIKAMI*2), NAOKI KIKUCHI*3), \\ HIROFUMI ZEMPO*1), HISASHI NAITO*1) \\ *1) Graduate School of Health and Sports Science, Juntendo University, Chiba, Japan, *2) Department of Sports and Life \\ Science, National Institute of Fitness and Sports in Kanoya, Kagoshima, Japan, *3) Sports Training Center, Nippon Sport \\ Science University, Tokyo, Japan
}

\begin{abstract}
A number of familial and twin studies have assessed the relative contribution of genetic and environmental factors to physical performance or its-related traits, and a significant genetic component has been predicted to affect phenotypes. A twin study indicated that the heritability of athletic status is $66 \%$. Thus, genetic factors appear to be important for determining elite sporting performance. To date, over 200 genes in both nuclear DNA and mitochondrial DNA (mtDNA) have been reported to be associated with physical performance and healthrelated fitness. However, most studies have been reported in European populations. In the Asian population, only 3 genetic loci have been linked to physical performance, including angiotensin I-converting enzyme and $\alpha$-actinin-3 genetic polymorphisms and mtDNA polymorphisms (mitochondrial haplogroups). In this review, we discuss the genetics of elite sporting performance, particularly in Asian populations, and their effects on lifestyle-related diseases such as type 2 diabetes and age-related muscle wasting (i.e., sarcopenia).
\end{abstract}

Key words: genetics, physical performance, type 2 diabetes, sarcopenia, Asian

\section{Introduction}

Within the field of sports science, elite sporting performance is understood to be the result of both nature (genetic factors) and nurture (environmental factors) such as physical training ${ }^{1)}$. A number of familial and twin studies have assessed the relative contribution of genetic and environmental factors to physical performance or its-related traits (e.g., maximum oxygen consumption, muscle strength, muscle power, and so on) and have estimated that there is a significant genetic component to these phenotypes. De Moor et al. ${ }^{2)}$ examined 4,488 British adult monozygotic and dizygotic female twins and estimated that the heritability of athletic status is $66 \%$. Thus, genetic factors appear to be important for determining elite sporting performance. Aerobic capacity appears to be affected more strongly by maternal inheritance than by paternal inheritance ${ }^{3)}{ }^{4)}$. This finding indicates that functional differences in maternally inherited mitochondrial DNA (mtDNA)-encoded proteins involved in oxidative phosphorylation affect aerobic performance. Therefore, mtDNA polymorphisms represent promising candidates influencing physical performance.

To date, numerous studies have identified genetic polymorphisms related to physical performance and its-related phenotypes. By 2009, more than 200 genes in both nuclear DNA and mtDNA had been reported to be associated with physical performance and health-related fitness ${ }^{5}$. The relationship between certain genetic polymorphisms and athletic performance was recently been reviewed ${ }^{6)-8}$. In these reviews, Ahmetov et al. indicated that at least 120 genetic variations are linked to elite athlete status based primarily on the candidate gene approach. These included 77 endurance

Corresponding author: Noriyuki Fuku

Graduate School of Health and Sports Science, Juntendo University

1-1 Hirakagakuendai, Inzai-shi, Chiba 270-1695, Japan

TEL: +81-476-98-1001 (ext. 9303) FAX: +81-476-98-1011 E-mail: noriyuki.fuku@nifty.com

The $2^{\text {nd }}$ Congress, International Academy of Sportology 〔Held on Sep. 12, 2015]

[Received Dec. 1, 2015] 
performance-related genetic variations and 43 sprint/power-related genetic makers from nuclear DNA and mtDNA. The number of genes associated with physical performance-related phenotypes is expected to increase dramatically with the application of genome-wide methods in elite athlete cohorts; however, few of these studies have been conducted. To date, only one genome-wide association study to identify genes associated with physical performance has been conducted, which was in Russia. Although numerous studies have been indicated an association between physical performance and genetic polymorphisms, most studies have examined the European population. In the Asian population, only 3 genetic loci are known to be linked to physical performance, including the angiotensin I-converting enzyme-ACE) and $\alpha$-actinin-3 (ACTN3) genetic polymorphisms and mtDNA polymorphisms (mitochondrial haplogroups). In this review, we discuss genetic studies of elite sporting performance, focusing on the Asian population, and their implications in lifestyle-related diseases such as type 2 diabetes and age-related muscle wasting (i.e., sarcopenia).

\section{Insertion/deletion polymorphism (rs4340) of the ACE gene}

ACE plays a critical role in circulatory homeostasis by causing blood vessels to constrict by converting angiotensin I to angiotensin II. This enzyme is also regulated in glucose metabolism by bradykinin, which induces muscle glucose uptake. In addition, local renin-angiotensin systems in a variety of tissues promote cell growth ${ }^{9}$. One of the ACE polymorphisms is a 287-base pair Alu repeat insertion/deletion (I/D) polymorphism (rs4340) in intron 16 , which was associated with circulating and tissue ACE levels. Higher ACE activity was associated with the $\mathrm{D}$ allele in both Caucasian populations and Asian populations, but not African populations.

A polymorphism in the ACE gene was first reported to impact on human physical performance in $1998^{10)}$. Many case-control association studies have reported that the ACE I/D polymorphism was associated with physical performance; particularly, I allele carriers showed lower ACE serum concentrations and greater success in endurance-related sporting performance ${ }^{10) 11)}$. In contrast, D allele carriers have higher ACE serum concentrations and have greater success in sprint/power-related sporting performance ${ }^{12)}$. Despite the consistency of such findings, data from East Asian populations have revealed conflicting results. Tobina et al. reported that the average running speeds of Japanese endurance and marathon runners with the DD+ID genotypes were significantly higher than those with the II genotype ${ }^{13)}$. In addition, Kim et al. reported that elite Korean power-oriented athletes with a higher frequency of the II+ID genotype had a higher probability of success in power-oriented sporting events ${ }^{14}$. Thus, opposite associations were observed between European and Asian populations. We investigated whether such associations differed by sporting event or by ethnicity, focusing on 200 elite Caucasian and 326 elite East Asian (158 Japanese and 168 Taiwanese)
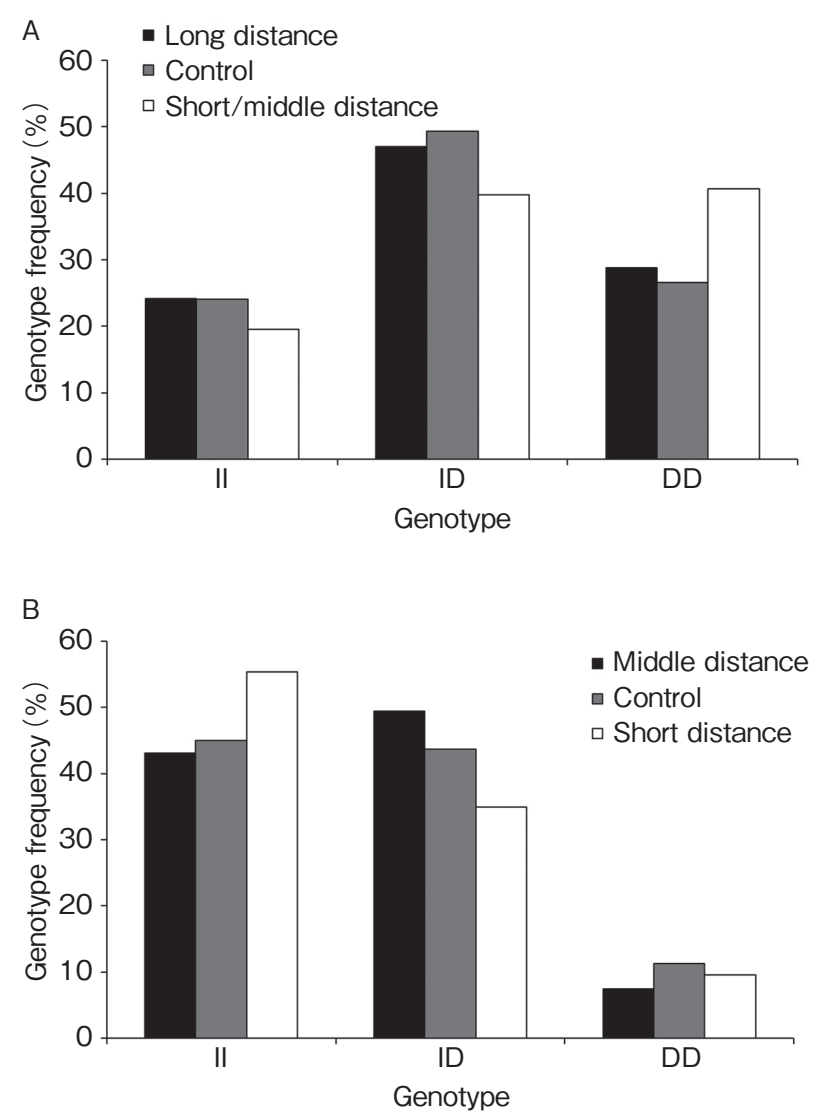

Figure-1 Genotype frequencies of ACE I/D polymorphism among elite Caucasian swimmers and controls (A) and elite East Asian swimmers and controls (B).

Modified from Wang G, et al: Med Sci Sports Exerc, 2013; 45: $892-900^{15)}$. 


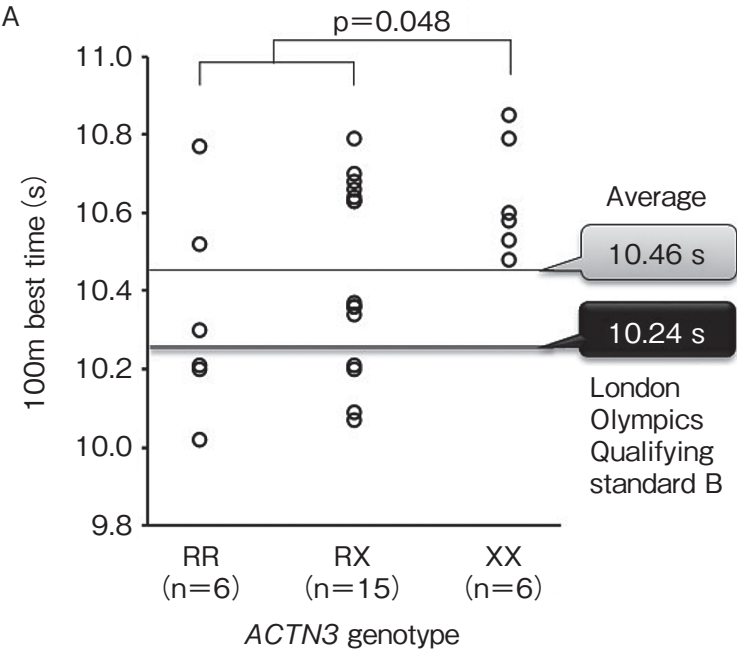

B

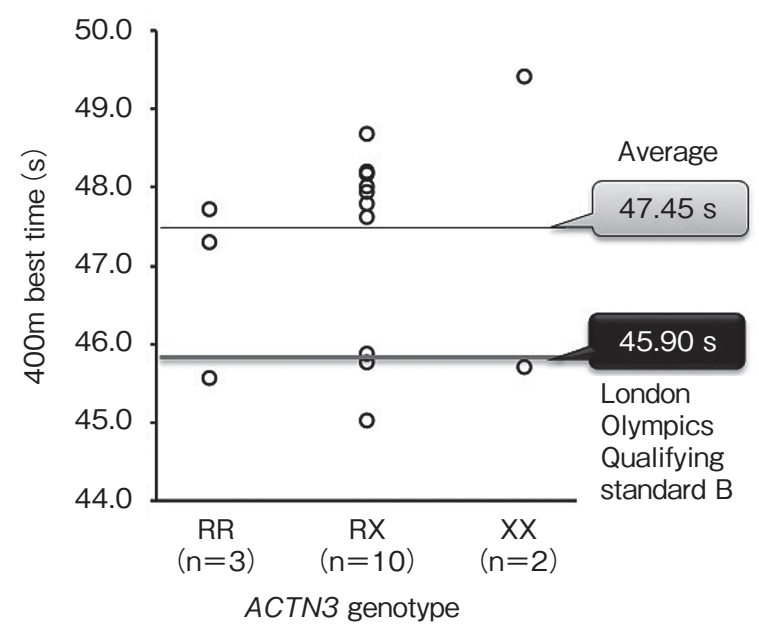

Figure-2 Associations of ACTN3 R577X polymorphism with 100-m (A) and 400-m (B) personal best times among male sprinters ${ }^{*} \mathrm{p}=0.042$.

Modified from Mikami E, et al: Int J Sports Med, 2014; 35: $172-177^{25)}$.

swimmers ${ }^{15)}$. In this study, the D allele was significantly associated with elite short/middle-distance swimmer status in Caucasians (Figure-1). However, the I allele was significantly associated with elite short-distance swimmer status in East Asians (Figure-2). We found that opposite alleles of the ACE I/D polymorphism were associated with elite sprint swimmer status in the Caucasian population and East Asian population. However, Kikuchi et $a l .{ }^{16)}$ reported that the DD genotype was associated with power-oriented performance. Further replicate and functional studies are necessary to confirm these findings.
It is well-known that the ACE I/D polymorphism is associated with lifestyle-related diseases in European and Asian populations, as this polymorphism shows the strongest association with circulating ACE activity in European and Asian populations but not African populations. The $\mathrm{D}$ allele of the ACE I/D polymorphism is a risk factor for type 2 diabetes in both European and Asian populations ${ }^{17)}{ }^{18)}$. Thus, physical performance-associated genetic polymorphisms, particularly ACE I/D, may be used to predict lifestyle-related diseases such as type 2 diabetes. This appears to be a reasonable hypothesis for European populations, as the I allele of the ACE I/D polymorphism may be an optimal factor affecting endurance performance because of the high prevalence of mitochondria-rich slowtwitch fibers, which are also associated with the resistance against to type 2 diabetes. However, this has not been confirmed in Asian populations.

\section{R577X polymorphism (rs1815739) of the ACTN3 gene}

$\alpha$-Actinin-2 and -3 proteins are localized to the Z-disk in the skeletal muscle and help to anchor actin filaments. $\alpha$-Actinin-2 is expressed in all human skeletal muscle fibers, whereas $\alpha$-actinin-3 is expressed only in human skeletal muscle fasttwitch fibers ${ }^{19)}$. A common genetic variation at codon 577 of the ACTN3 gene causes the amino acid replacement of the arginine $(\mathrm{R})$ with a stop codon (X), i. e., R577X. Homozygosity for the common nonsense polymorphism R577X in the ACTN3 gene results in complete deficiency of $\alpha$-actinin-3 in fast-twitch muscle fibers. Experiments in knockout mice showed that ACTN3 deficiency affects skeletal muscle function ${ }^{20)}$.

As described above, more than 200 genetic variants have been associated with physical performance; the most widely studied variant is the ACTN3 R577X polymorphism. Yang et al. ${ }^{21)}$ reported that $\mathrm{R}$ allele carriers ( $\mathrm{RR}+\mathrm{RX}$ genotype) of this R577X polymorphism was found more frequently in elite Australian sprint/power athletes than in controls. This finding has been replicated in a broad variety of ethnic groups (See review of Ahmetov et al.). In a meta-analysis, Alfred et al. ${ }^{22)}$ found that the ACTN3 RR genotype was more common among European sprint/power athletes 
than among controls. Another meta-analysis reported a positive association between the ACTN3 $\mathrm{RR}+\mathrm{RX}$ genotype and sprint/power athletic status in Europeans, but not in Asian and African populations ${ }^{23)}$. Thus, the association between ACTN3 R577X and elite Asian sprint/power athlete status was largely unknown until recently.

We recently investigated the association between the ACTN3 R577X polymorphism and elite Japanese athlete status in a large cohort of Japanese track and field athletes $(\mathrm{n}=627$ for sprint/power athlete and 430 for endurance athletes) and controls $(\mathrm{n}=810)^{24)}$. We found that sprinters with the RR+ RX genotype had faster personal best times for the 100-m event than those with the XX genotype (Figure-2A ${ }^{25)}$. Interestingly, all 7 male sprinters who had achieved the London 2012 Olympic qualifying standard for the $100-\mathrm{m}$ event-B standard: $10.24 \mathrm{~s}$ ) possessed the RR or RX genotypes (Figure-2A), which was not observed in those qualifying for the $400-\mathrm{m}$ event (Figure-2B) ${ }^{25}$. Our results indicates that sprinters with the $\mathrm{XX}$ genotype may experience difficulty in the 100-m event and that athletes with the XX genotype transfer may perform better in other events (e.g., long jump or $400-\mathrm{m}$ event). Interestingly, the ACTN3 R577X polymorphism accounted for $12 \%$ of the variability in the personal best times for the 100-m run.

It was previously reported that the ACTN3 R577X polymorphism is associated with muscle fiber composition ${ }^{26)}$ and muscle strength ${ }^{27)}{ }^{28)}$. We previously reported that older women with the XX genotype had a smaller thigh-muscle cross-sectional area than those with the RR and RX genotypes, but this was not the case in middle-aged women ${ }^{29)}$. This difference appears to be equivalent to 10 years of aging-related muscle wasting. A major characteristic of sarcopenia is the specific loss of type II muscle fibers ${ }^{30}$. Therefore, loss of the ACTN3 protein in type II muscle fiber may be associated with sarcopenia. Delmonico et al. ${ }^{31)}$ conducted a longitudinal study for 5 years and found that the XX genotype had a greater risk of incident lower extremity limitation compared to the RR and RX genotypes in elderly women. Thus, this polymorphism may affect health-related muscle fitness (e.g., sarcopenia) as well as sprint/power performance.

\section{Haplogroups of mtDNA}

Mitochondria are essential to all higher organisms for sustaining life and are extremely important in energy metabolism, providing 36 molecules of ATP per glucose molecule in contrast to the 2 ATP molecules produced by glycolysis. It is reasonable to hypothesize that mitochondria play an important role in determining aerobic performance, as mitochondria supply the majority of cellular ATP through oxidative phosphorylation (OXPHOS), which is the main source of energy for endurance exercise.

Although most DNA is packaged in chromosomes within the nucleus, mitochondria also possess their own circular DNA, designated as mtDNA. The 16,569-base pair human mtDNA contains 13 genes for mitochondrial OXPHOS, as well as 2 rRNA and 22 tRNA genes necessary for protein synthesis within mitochondria. Therefore, the diversity of mtDNA may affect elite endurance athlete status. Since polymorphisms in mtDNA are linked with other mtDNA polymorphisms, many studies have been reported the association between mitochondrial haplogroups, a set of tightly linked mtDNA polymorphisms, and physical performance.

It is important to examine the association between phenotypic traits and mtDNA polymorphisms in terms of population, ethnicity, and/or race, because mtDNA sequences, particularly those in the control region, are known to vary among populations. We sequenced hypervariable sequence 1 , a part of control region, and the $\mathrm{C}>\mathrm{A}$ polymorphism at m. 5178 within the ND2 gene of the mtDNA. We then compared the percent frequency of mitochondrial haplogroups in 141 Japanese Olympians ( $n=60$ for sprint/power athletes and $n=$ 81 for endurance athletes) with 672 Japanese controls ${ }^{32)}$. Subjects were classified into 12 major Japanese mitochondrial haplogroups (F, B, A, N9a, N9b, M7a, M7b, M*, G2, G1, D5, or D4). The distribution of mitochondrial haplogroups in endurance athletes and sprint/power athletes relative to that in the controls is shown in Figure-3. We found that endurance athletes displayed an excess of haplogroup G1. In contrast, sprint/power athletes showed a greater proportion of haplogroup F. We evaluated the detailed characteristics of mtDNA polymorphisms and mitochondrial haplogroups in 


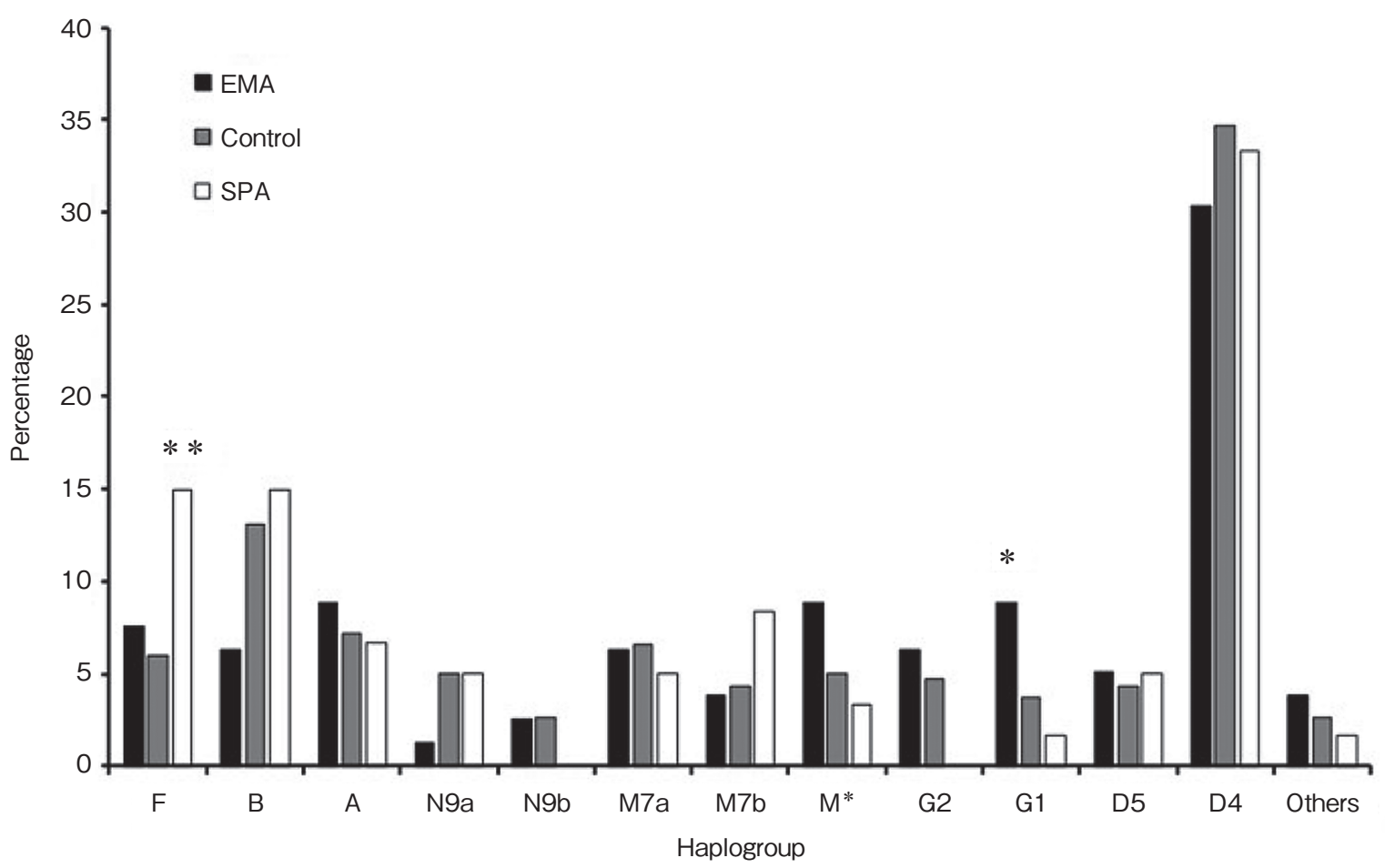

Figure-3 Mitochondrial haplogroup distribution among endurance/middle power athletes, sprint/power athletes, and controls

Significant differences compared to Controls (gray bars) are indicated by asterisks $\left({ }^{*} \mathrm{p}<0.05,{ }^{* *} \mathrm{p}<0.01\right)$. For haplogroup analysis, we compared each haplogroup versus the sum of all other haplogroups. $\mathrm{P}$ value $<0.05$ was considered statistically significant. EMA: endurance/middle-power athletes, SPA: sprint/power athletes.

Modified from Mikami E, et al: Br J Sports Med, 2011; 45: 1179-1183 ${ }^{32}$.

elite Japanese athletes and found that some were associated with sprint/power performance as well as endurance performance ${ }^{33)}$ (34).

Although numerous studies have reported associations between aerobic performance phenotypes and mitochondrial haplogroups, studies on the associations between "anaerobic" performance phenotypes and mitochondrial haplogroups are limited. Because "anaerobic" capacity relies more heavily upon glycolysis than mitochondrial OXPHOS, mitochondrial haplogroups are not thought to be related to anaerobic capacity. However, we found a positive association between mitochondrial haplogroup $\mathrm{F}$ and sprint/power performance in the Japanese population. We also examined the effect of mitochondrial haplogroups on anaerobic performance phenotypes such as muscle power, muscle strength, and muscle mass in 480 healthy Japanese non-athlete adults, and found that macrohaplogroup $\mathrm{N}$ was significantly associated with stronger leg extension power and higher vertical jump performance compared with macroha- plogroup $\mathrm{M}$ for these Japanese subjects ${ }^{35}$. Mitochondrial haplogroup $\mathrm{F}$, which is associated with elite Japanese SPA status, is a major component of macrohaplogroup $\mathrm{N}^{36}$, which is associated with muscle power in non-athletic Japanese individuals.

We previously reported that mitochondrial haplogroups were associated with type 2 diabetes in Asian populations ${ }^{37}$. Particularly, mtDNA haplogroup N9a was associated with a decreased risk of type 2 diabetes, whereas haplogroups $\mathrm{F}$ and D5 were associated with an increased risk of type 2 diabetes. Thus, mitochondrial haplogroup $\mathrm{F}$ was associated with not only sprint/power performance, but also type 2 diabetes. Hwang et al. ${ }^{38)}$ reported interesting results regarding the association between mitochondrial haplogroups and gene expression profiles in transmitochondrial hybrid cells. Based on the gene expression data from the hybrid cell with haplogroups $\mathrm{F}$ and N9a, haplogroup $\mathrm{F}$ showed lower gene expression of the oxidative phosphorylation pathway compared with haplogroup N9a. In contrast, haplogroup F showed 
greater expression of glycolysis pathway genes compared with N9a. Thus, mitochondrial DNA polymorphisms influence outer mitochondrial functions as well as inner mitochondrial functions.

\section{Future directions}

In this article, we mainly discussed the genetics of sporting performance and its implications on lifestyle-related diseases and/or age-related muscle wasting (i.e., sarcopenia). Particularly, we discussed these findings in Asian (Japanese) subjects. Numerous studies have attempted to identify genetic polymorphisms associated with physical performance, but few studies have been conducted in Asian populations. Therefore, it is necessary to validate the association between the genetic polymorphisms and athletic status, sprint/power performance, and/or endurance performance in the Asian population. Furthermore, subjects in some previous case-control studies were athletes participating in various events, making it difficult to compare the results.

Current studies of athletic performance-associated polymorphisms, which were previously identified through candidate gene-association studies, have revealed the polygenic profile for determining athletic performance ${ }^{39)}$;0); however, additional studies are needed to explain the genetic component. The strong genetic contributions to physical performance and/or its-related traits indicate the possibility of using genetic approaches to individualize training approaches for enhancing competitive abilities in sports or even to help select appropriate athletic events by using genetic screening for extending the personal limit of athletes (i.e., talent identification and/or talent transfer to other events). Further studies in sports science should include more detailed analyses of genetic polymorphisms detected in both mtDNA and nuclear DNA, including whole genome sequencing for mtDNA and genome-wide association studies for nuclear $\mathrm{DNA}^{41)}$. Some polymorphisms, which are found form whole genome wide approach using elite athletes, have a potential to identify novel polymorphisms for determining health-related phenotypes such as type 2 diabetes and/or sarcopenia.

\section{References}

1) Tucker R, Collins M: What makes champions? A review of the relative contribution of genes and training to sporting success. Br J Sports Med, 2012; 46: 555-561.

2) De Moor MH, Spector TD, Cherkas LF, et al: Genome-wide linkage scan for athlete status in 700 British female DZ twin pairs. Twin Res Hum Genet, 2007; 10: 812-820.

3) Bouchard C, An P, Rice T, et al: Familial aggregation of VO (2max) response to exercise training: results from the HERITAGE Family Study. J Appl Physiol, 1999; 87: 1003-1008.

4) Lesage R, Simoneau JA, Jobin J, et al: Familial resemblance in maximal heart rate, blood lactate and aerobic power. Hum Hered, 1985; 35: 182-189.

5) Bray MS, Hagberg JM, Perusse L, et al: The human gene map for performance and health-related fitness phenotypes: the 2006-2007 update. Med Sci Sports Exerc, 2009; 41: 35-73.

6) Ahmetov II, Fedotovskaya ON: Current progress in sports genomics. Adv Clin Chem, 2015; 70: 247-314.

7) Fuku N, Mikami E, Tanaka M: Association of mitochondrial DNA polymorphisms and/or haplogroups with elite Japanese athlete status. J Phys Fitness Sports Med, 2013; 2: 17-27.

8) Wang G, Padmanabhan S, Wolfarth B, et al: Genomics of elite sporting performance: what little we know and necessary advances. Adv Genet, 2013; 84: 123-149.

9) Puthucheary Z, Skipworth JR, Rawal J, et al: The ACE gene and human performance: 12 years on. Sports Med, 2011; 41: 433-448.

10) Montgomery HE, Marshall $\mathrm{R}$, Hemingway $\mathrm{H}$, et al: Human gene for physical performance. Nature, 1998; 393: 221-222.

11) Myerson S, Hemingway $\mathrm{H}$, Budget $\mathrm{R}$, et al: Human angiotensin I-converting enzyme gene and endurance performance. J Appl Physiol (1985), 1999; 87: 13131316.

12) Costa AM, Silva AJ, Garrido ND, et al: Association between ACE D allele and elite short distance swimming. Eur J Appl Physiol, 2009; 106: 785-790.

13) Tobina T, Michishita R, Yamasawa F, et al: Association between the angiotensin I-converting enzyme gene insertion/deletion polymorphism and endurance running speed in Japanese runners. J Physiol Sci, 2010; 60: 325-330.

14) Kim CH, Cho JY, Jeon JY, et al: ACE DD genotype is unfavorable to Korean short-term muscle power athletes. Int J Sports Med, 2010; 31: 65-71.

15) Wang G, Mikami E, Chiu LL, et al: Association analysis of ACE and ACTN3 in elite Caucasian and East Asian swimmers. Med Sci Sports Exerc, 2013; 45: 892-900.

16) Kikuchi N, Min SK, Ueda D, et al: Higher frequency of the ACTN3 R allele + ACE DD genotype in Japanese elite wrestlers. J Strength Cond Res, 2012; 26: 32753280 .

17) Daimon M, Oizumi T, Saitoh T, et al: The D allele of the angiotensin-converting enzyme insertion/deletion-I/D) polymorphism is a risk factor for type 2 diabetes in a population-based Japanese sample. Endocr J, 2003; 50: 393-398.

18) Stephens JW, Dhamrait SS, Cooper JA, et al: The D allele of the ACE I/D common gene variant is associated 
with Type 2 diabetes mellitus in Caucasian subjects. Mol Genet Metab, 2005; 84: 83-89.

19) North KN, Beggs AH: Deficiency of a skeletal muscle isoform of alpha-actinin (alpha-actinin-3) in merosinpositive congenital muscular dystrophy. Neuromuscul Disord, 1996; 6: 229-235.

20) Berman Y, North KN: A gene for speed: the emerging role of alpha-actinin-3 in muscle metabolism. Physiology (Bethesda), 2010; 25: 250-259.

21) Yang N, MacArthur DG, Gulbin JP, et al: ACTN3 genotype is associated with human elite athletic performance. Am J Hum Genet, 2003; 73: 627-631.

22) Alfred T, Ben-Shlomo Y, Cooper R, et al: ACTN3 genotype, athletic status, and life course physical capability: meta-analysis of the published literature and findings from nine studies. Hum Mutat, 2011; 32: 10081018.

23) Ma F, Yang Y, Li X, et al: The association of sport performance with ACE and ACTN3 genetic polymorphisms: a systematic review and meta-analysis. PLoS One, 2013; 8: e54685.

24) Kikuchi N, Miyamoto-Mikami E, Murakami H, et al: ACTN3 R577X genotype and athletic performance in a large cohort of Japanese athletes. Eur J Sport Sci, 2015: 1-8. [Epub ahead of print]

25) Mikami E, Fuku N, Murakami H, et al: ACTN3 R577X genotype is associated with sprinting in elite Japanese athletes. Int J Sports Med, 2014; 35: 172-177.

26) Vincent B, Windelinckx A, Nielens $H$, et al: Protective role of alpha-actinin-3 in the response to an acute eccentric exercise bout. J Appl Physiol, 2010; 109: 564573.

27) Clarkson PM, Devaney JM, Gordish-Dressman H, et al: ACTN3 genotype is associated with increases in muscle strength in response to resistance training in women. J Appl Physiol, 2005; 99: 154-163.

28) MacArthur DG, Seto JT, Chan S, et al: An Actn3 knockout mouse provides mechanistic insights into the association between alpha-actinin-3 deficiency and human athletic performance. Hum Mol Genet, 2008; 17: 1076-1086

29) Zempo H, Tanabe K, Murakami H, et al: Age differences in the relation between ACTN3 R577X polymorphism and thigh-muscle cross-sectional area in women. Genet Test Mol Biomarkers, 2011; 15: 639-643.
30) Lexell J: Human aging, muscle mass, and fiber type composition. J Gerontol A Biol Sci Med Sci, 1995; 50 Spec No: $11-16$.

31) Delmonico MJ, Zmuda JM, Taylor BC, et al: Association of the ACTN3 genotype and physical functioning with age in older adults. J Gerontol A Biol Sci Med Sci, 2008; 63: $1227-1234$.

32) Mikami E, Fuku N, Takahashi H, et al: Mitochondrial haplogroups associated with elite Japanese athlete status. Br J Sports Med, 2011; 45: 1179-1183.

33) Mikami E, Fuku N, Kong QP, et al: Comprehensive analysis of common and rare mitochondrial DNA variants in elite Japanese athletes: a case-control study. J Hum Genet, 2013; 58: 780-787.

34) Mikami E, Fuku N, Takahashi H, et al: Polymorphisms in the control region of mitochondrial DNA associated with elite Japanese athlete status. Scand J Med Sci Sports, 2013; 23: 593-599.

35) Fuku N, Murakami H, Iemitsu M, et al: Mitochondrial macrohaplogroup associated with muscle power. Int J Sports Med, 2012; 33: 410-414.

36) Tanaka M, Cabrera VM, Gonzalez AM, et al: Mitochondrial genome variation in eastern Asia and the peopling of Japan. Genome Res, 2004; 14: 1832-1850.

37) Fuku N, Park KS, Yamada Y, et al: Mitochondrial haplogroup N9a confers resistance against type 2 diabetes in Asians. Am J Hum Genet, 2007; 80: 407-415.

38) Hwang S, Kwak SH, Bhak J, et al: Gene expression pattern in transmitochondrial cytoplasmic hybrid cells harboring type 2 diabetes-associated mitochondrial DNA haplogroups. PLoS One, 2011; 6: e22116.

39) Santiago C, Ruiz JR, Muniesa CA, et al: Does the polygenic profile determine the potential for becoming a world-class athlete? Insights from the sport of rowing. Scand J Med Sci Sports, 2010; 20: e188-194.

40) Williams AG, Folland JP: Similarity of polygenic profiles limits the potential for elite human physical performance. J Physiol, 2008; 586: 113-121.

41) Bouchard C, Sarzynski MA, Rice TK, et al: Genomic predictors of the maximal $\mathrm{O}$ (2) uptake response to standardized exercise training programs. J Appl Physiol, 2011; 110: 1160-1170. 